\title{
Advances in antibody therapeutics targeting small-cell lung cancer
}

\author{
Hongyang $\mathrm{Lu}^{1,2, \mathrm{~A}-\mathrm{F}}$, Zhiming Jiang ${ }^{1, B, D}$ \\ ${ }^{1}$ Zhejiang Key Laboratory of Diagnosis and Treatment Technology on Thoracic Oncology (Lung and Esophagus), Zhejiang Cancer Hospital, Hangzhou, China \\ 2 Department of Thoracic Medical Oncology, Zhejiang Cancer Hospital, Hangzhou, China \\ A - research concept and design; $\mathrm{B}$ - collection and/or assembly of data; $\mathrm{C}$ - data analysis and interpretation; \\ $D$ - writing the article; $E$ - critical revision of the article; $F$ - final approval of the article
}

\section{Address for correspondence \\ Hongyang Lu \\ E-mail: luhy@zjcc.org.cn \\ Funding sources \\ This work was supported by the Zhejiang Provincial Natural Science Foundation of China (No. LY15H290001), Public Welfare Technology Application Studies Program of the Zhejiang Province (No. 2016C33118) and the 1022 Talent Training Program of Zhejiang Cancer Hospital.}

Conflict of interest

None declared

Received on July 13, 2016

Reviewed on November 17, 2017

Accepted on April 3, 2017

\begin{abstract}
The proportion of small-cell lung cancer (SCLC) among all lung cancers decreased from 17.26\% in 1986 to $12.95 \%$ in 2002 . Chemotherapy is the key mode of treatment. However, novel therapeutic strategies and drugs are imperative, as the prognosis remains poor. In recent years, antibody therapies have shown promising prospects against malignancy. This review focuses on the advances in antibody therapies in SCLC. Although the results of pembrolizumab, nivolumab, ipilimumab, and rovalpituzumab tesirine are inspiring, all of the clinical trials on these drugs are phase I/II and have been verified for further phase III clinical trials. It was demonstrated that chemotherapy in combination with bevacizumab can improve the progression-free survival (PFS) in phase III trials. The insulin-like growth factor-1 receptor (IGF-1R) is associated with a poor prognosis in SCLC, while the anti-IGF-1R monoclonal antibody figitumumab has a potential therapeutic value. Tarextumab, an antibody that blocks both Notch2 and Notch3 signaling, in combination with etoposide and platinum (EP) in patients with untreated extensive-stage SCLC, proved to be well-tolerated and showed dosedependent anti-tumor activity. The therapeutic effect of sacituzumab govitecan, BW-2 and lorvotuzumab mertansine in SCLC warranted further evaluation. Bec2/BCG as an adjuvant vaccination in patients with limited-disease SCLC could not improve the survival, PFS, or quality of life. Thus, clinical studies are essential to confirm the anti-tumor efficacy of trastuzumab in SCLC.
\end{abstract}

Key words: small-cell lung cancer, antibody therapeutics, antibody-drug conjugate

D0I

10.17219/acem/70159

\section{Copyright}

Copyright by Author(s)

This is an article distributed under the terms of the

Creative Commons Attribution Non-Commercial License

(http://creativecommons.org/licenses/by-nc-nd/4.0/) 
Lung cancer, including small-cell lung cancer (SCLC) and non-small-cell lung cancer (NSCLC), is the leading cause of cancer-related mortality and the most common malignancy in China as well as around the world. ${ }^{1,2}$ The proportion of SCLC among all lung cancers decreased from $17.26 \%$ in 1986 to $12.95 \%$ in $2002 .{ }^{3}$ Chemotherapy is the primary mode of treatment of SCLC; however, the prognosis still remains poor. To date, no targeted drugs for SCLC have been approved by the US Food and Drug Administration (FDA). Thus, novel therapeutic strategies and drugs are imperative. In recent years, antibody therapies have shown promising anti-tumor prospects in a variety of human cancers. The present review focuses on the advances in antibody therapies in SCLC, which include pembrolizumab, nivolumab, ipilimumab, bevacizumab, rovalpituzumab tesirine, sacituzumab govitecan, and lorvotuzumab mertansine (IMGN901).

\section{Immune checkpoint inhibitors}

Cytotoxic T-lymphocyte-associated protein 4 (CTLA-4) and programmed cell death-1 (PD-1) are typical immune checkpoint molecules that suppress anti-tumor immunity. Antibodies that keep the PD-1 and CTLA-4 immune checkpoint pathways as targets display superior clinical efficacy. It was assumed that pembrolizumab and nivolumab (anti-PD-1 antibody), as well as ipilimumab (anti-CTLA-4 antibody), act with the most promising immunotherapies under investigation in SCLC patients. ${ }^{4}$ Pembrolizumab is a humanized immunoglobulin G4 (IgG4) monoclonal antibody against PD-1 designed to block the interaction between PD-1 and its ligands, programmed cell death ligand-1 (PD-L1) and programmed cell death ligand-2 (PD-L2). Pembrolizumab has demonstrated robust anti-tumor activity and a manageable toxicity profile in NSCLC. The safety and efficacy of pembrolizumab was assessed in PD-L1-positive SCLC patients in a multicohort, phase 1b KEYNOTE-028 study. ${ }^{5}$ The results of this study demonstrated that the SCLC patients either did not respond to or were unable to receive the standard therapy. The authors observed the PD-L1 expression in $\geq 1 \%$ of cells in tumor nests or PD-L1-positive bands in stroma, as assessed by immunohistochemistry (IHC), using 22C3 anti-PD-L1 antibody. Pembrolizumab $(10 \mathrm{mg} / \mathrm{kg})$ was administered every 2 weeks for up to 2 years or until confirmed progression or unacceptable toxicity. Of the 147 SCLC patients with evaluable tumor samples screened for the PD-L1 expression, 42 patients (29\%) showed PD-L1-positive tumors. Overall, 24 patients with SCLC were enrolled in the study and received at least 1 pembrolizumab dose. All 24 experienced drug-related adverse events (AEs) and only 2 patients showed grade 3 or higher drug-related AEs. On the other hand, 1 patient had a complete response (CR), and 7 patients had partial response (PR). The median progressionfree survival rate (PFS) was 1.9 months, with a 6-month PFS rate of $28.6 \% .^{5}$ This study was only a phase $1 \mathrm{~b}$ trial with a limited sample size, so further investigation in randomized, controlled clinical trials is needed. Pembrolizumab is well-tolerated and promising in SCLC patients.

The safety and activity of nivolumab and nivolumab plus ipilimumab in patients with SCLC who progressed after 1 or more previous regimens were assessed in a multicenter, multi-arm, open-label phase $1 / 2$ trial. A total of 216 patients were enrolled and received nivolumab ( $3 \mathrm{mg} / \mathrm{kg}) \mathrm{ev}-$ ery 2 weeks (until disease progression or unacceptable toxicity) or nivolumab plus ipilimumab (1 mg/ $\mathrm{kg}$ plus $1 \mathrm{mg} / \mathrm{kg}$, $1 \mathrm{mg} / \mathrm{kg}$ plus $3 \mathrm{mg} / \mathrm{kg}$, or $3 \mathrm{mg} / \mathrm{kg}$ plus $1 \mathrm{mg} / \mathrm{kg}$, intravenously) every 3 weeks for 4 cycles, followed by $3 \mathrm{mg} / \mathrm{kg}$ of nivolumab every 2 weeks. The primary endpoint was an objective response rate (ORR). An objective response was achieved in 10 of 98 patients (10\%) receiving $3 \mathrm{mg} / \mathrm{kg}$ of nivolumab, in 1 of 3 patients (33\%) receiving $1 \mathrm{mg} / \mathrm{kg}$ of nivolumab plus $1 \mathrm{mg} / \mathrm{kg}$ of ipilimumab, in 14 of $61 \mathrm{pa}-$ tients (23\%) receiving $1 \mathrm{mg} / \mathrm{kg}$ of nivolumab plus $3 \mathrm{mg} / \mathrm{kg}$ of ipilimumab, and in 10 of 54 patients (19\%) receiving $3 \mathrm{mg} / \mathrm{kg}$ of nivolumab plus $1 \mathrm{mg} / \mathrm{kg}$ of ipilimumab. Grade 3 or 4 treatment-related AEs occurred in 13 patients (13\%) in the $3 \mathrm{mg} / \mathrm{kg}$ nivolumab cohort, in $18(30 \%)$ in the $1 \mathrm{mg} / \mathrm{kg}$ nivolumab plus $3 \mathrm{mg} / \mathrm{kg}$ ipilimumab cohort and in 10 (19\%) in the $3 \mathrm{mg} / \mathrm{kg}$ nivolumab plus $1 \mathrm{mg} / \mathrm{kg}$ ipilimumab cohort. The most commonly reported grade 3 or 4 treatment-related AEs were increased lipase and diarrhea. Two patients who received $1 \mathrm{mg} / \mathrm{kg}$ of nivolumab plus $3 \mathrm{mg} / \mathrm{kg}$ of ipilimumab died due to treatment-related AEs, such as myasthenia gravis and aggravated renal failure, and 1 patient who received $3 \mathrm{mg} / \mathrm{kg}$ of nivolumab plus $1 \mathrm{mg} / \mathrm{kg}$ of ipilimumab died from treatment-related pneumonitis. Toxicity and efficacy, as well as safety profiles, should be carefully weighed. These results demonstrated a similar response to pembrolizumab. ${ }^{5,6}$ This data supports the evaluation of nivolumab and nivolumab plus ipilimumab in another phase 3 randomized, controlled trials in SCLC after first-line platinum-based chemotherapy or maintenance therapy. ${ }^{6}$ These results demonstrated a similar response to pembrolizumab as in the case of nivolumab plus ipilimumab in relapsed SCLC patients; the question which therapy is superior remains unanswered.

Tumor-infiltrating lymphocytes and the PD-L1 expression are frequently observed in brain metastases of SCLC. The presence of CD45RO+ memory T cells in SCLC brain metastases seems to be associated with favorable survival times. SCLC brain metastases exhibit an active immune microenvironment which might be targetable by immunemodulating drugs. ${ }^{7}$ PD-L1 protein was infrequently expressed in SCLC (7.3\%) and correlated with the limited disease stage that might serve as a prognostic marker for enhanced overall survival (OS). ${ }^{8,9}$ The PD-L1 expression in 40 surgically resected SCLC specimens was evaluated by IHC with 3 different antibodies - E1L3N, 28-8 and SP142 clones - and using 3 different evaluations: all red score, $1 \%$ cut-off and $5 \%$ cut-off. The IHC evaluations with 
the 5\% cut-off showed similar rates of expression, using the 3 different antibodies. ${ }^{10}$ The reagents and methods of PDL-1 detection require further standardization, and the cut-off value still needs to be consolidated.

\section{Bevacizumab}

Tumor growth and nutrition for angiogenesis is mediated primarily via the vascular endothelial growth factor (VEGF) family. The inhibition of angiogenesis has been the subject of research and clinical investigation. Bevacizumab is an anti-VEGF monoclonal antibody that inhibits angiogenesis and has been approved as the first-line treatment for advanced NSCLC. ${ }^{11}$ According to the study by Ustuner et al., low serum VEGF concentration is a significant and independent prognostic factor in SCLC patients; however, the surveillance of VEGF and its receptors was not useful in predicting chemotherapy response. ${ }^{12}$ A randomized phase $2 / 3$ trial, which evaluated the efficacy and safety of bevacizumab added following the induction of chemotherapy in extensive-stage SCLC, demonstrated no difference in the response or PFS after randomization. Furthermore, serum VEGF and soluble VEGF receptor titrations failed to identify predictive biomarkers. ${ }^{13}$ An Italian multicenter, randomized phase 3 study of cisplatin-etoposide (EP) with or without bevacizumab as the first-line treatment in extensive-stage SCLC showed that the addition of bevacizumab led to a statistically significant improvement in PFS (5.7 vs 6.7 months, hazard ratio (HR): 0.72; 95\% confidence interval (CI): 0.54-0.97; $\mathrm{p}=0.030)$ and an acceptable toxicity profile. However, a statistically insignificant increase in the primary endpoint of OS was observed. In this trial, patients were randomized to receive either $100 \mathrm{mg} / \mathrm{m}^{2}$ of etoposide intravenously (iv.) and $25 \mathrm{mg} / \mathrm{m}^{2}$ of cisplatin on days 1-3 (or carboplatin AUC 5 on day 1) (group A), or the same chemotherapy combined with $7.5 \mathrm{mg} / \mathrm{kg}$ of bevacizumab iv. on day 1 (group B) every 3 weeks for a maximum of 6 courses. In the absence of progression after 6 cycles, patients in group B continued bevacizumab alone until progression or for a maximum of 18 courses. ${ }^{14}$ An EP combined with bevacizumab regimen followed by oral etoposide and bevacizumab maintenance treatment appeared to be feasible and efficient in terms of the 9-month disease control rate (36.3\%) in patients with extensive-stage SCLC; severe AEs were rarely observed during the maintenance treatment. Since this data comes from a single institution with only 22 patients in the study, further clinical trials are essential to confirm the result. ${ }^{15}$ Another study which involved the addition of bevacizumab to paclitaxel showed no improvement in the outcomes of 34 relapsed chemosensitive SCLC patients. The median PFS was recorded as 14.7 weeks. ${ }^{16}$ This combination was feasible and active in chemoresistant relapsed SCLC as a salvage treatment in 30 patients with a median PFS of 2.7 months, which represented a valid therapeutic alternative. However, further evaluation is imperative. ${ }^{17}$ The predictive factors for the therapeutic effect of bevacizumab are unclear, and which types of patients can benefit from the use of bevacizumab is still unknown. The US FDA has not approved bevacizumab for the treatment of SCLC. The selection of appropriate SCLC patients for a bevacizumab therapy is an important area of research.

\section{Anti-insulin-like growth factor-1 receptor monoclonal antibody}

The activation of the insulin-like growth factor-1 receptor (IGF-1R) - an autocrine growth factor - by IGF-1 and IGF-2 plays a key role in the growth of malignant tumors and the inhibition of apoptosis. The IGF-1R pathway is generally upregulated, and the IGF-1R inhibition is a plausible therapeutic strategy in SCLC. The prognosis of SCLC patients is poor, as assessed by $3+$ immunostaining of IGF-1R. ${ }^{18}$ The addition of R1 507 - a monoclonal antibody against IGF-1R to the current standard cisplatinionizing radiation doublet revealed remarkable chemo- and radiosensitizing effects in selected SCLC models, and warrants further investigations in clinical settings. ${ }^{19} \mathrm{Cao}$ et al. showed that the IGF-1R expression was significantly associated with tumor size, node (N) status, stage, and Ki-67, and the multivariate Cox analysis demonstrated that only the IGF-1R expression was found to be an independent predictor for OS. The study suggested that the IGF-1R expression was negatively correlated with the survival of patients. Additionally, anti-IGF-1R monoclonal antibody figitumumab exhibited a potential therapeutic effect through the IGF-1R blockage and downregulation. ${ }^{20}$ The anti-IGF-1R monoclonal antibody exhibits anti-tumor activity in SCLC. The anti-IGF-1R therapeutic approach needs to be further investigated in clinical trials.

\section{Tarextumab and MEDI0639}

The canonical Notch pathway with 4 Notch receptors (Notch1-4) and 5 ligands (DLL1, 3-4, Jagged1-2) is an evolutionarily conserved cell signaling pathway that plays a prominent role in the determination of cell fate, differentiation, proliferation, and death. Several drugs that targeted the Notch pathway for various malignant tumors have been developed. ${ }^{21}$ Tarextumab is an antibody which blocks both Notch 2 and Notch 3 signaling pathways and has been confirmed to inhibit tumor growth in patient-derived xenograft tumors. ${ }^{22}$ A phase $1 b$ study of tarextumab in combination with EP in untreated extensive-stage SCLC patients showed that this combination was well-tolerated with dose-dependent anti-tumor activity. Twenty-seven patients were treated with tarextumab doses ranging from 5 to $15 \mathrm{mg} / \mathrm{kg}$. However, the maximum tolerated dose (MTD) was not reached and it was determined that 
$15 \mathrm{mg} / \mathrm{kg}$ of tarextumab was a phase 2 dose. The results showed that the ORR was $73 \%$ and that the median OS was 10.3 months with a median follow-up of 9.8 months; 16.0 months for patients receiving $\geq 12.5 \mathrm{mg} / \mathrm{kg}$ of tarextum$\mathrm{ab}$ and 7.6 months for patients with a dosage $<12 \mathrm{mg} / \mathrm{kg} .{ }^{23}$ Frequently reported $(\geq 15 \%)$ tarextumab-related AEs were mostly grade 1 or 2 , they were also reversible. A randomized, placebo-controlled phase 2 study with this regimen is ongoing. The appropriate dosage of tarextumab requires further exploration.

MEDI0639 is a human monoclonal antibody that selectively binds to DLL4 and inhibits DLL4-Notch receptor signaling. Preclinical studies demonstrated that the DLL4 blockade can reduce tumor cell proliferation and cancer stem cell frequency. A single-agent phase 1 study of MEDI0639 on 9 SCLC patients suggested that MEDI0639 may suppress cancer stem cell counts and activities. ${ }^{24}$ However, further studies are required to evaluate the effect of MEDI0639 in a significant number of patients in a phase 1 study.

\section{Tucotuzumab}

Tucotuzumab - a humanized KS-interleukin-2 and an EpCAM-specific immune cytokine - combined with cyclophosphamide showed immunological activity. A randomized, open-label phase 2 study compared tucotuzumab/cyclophosphamide as maintenance therapy with the best supportive care in extensive-disease SCLC patients who responded to the first-line platinum-based chemotherapy, but the results showed no PFS or OS benefits. ${ }^{25}$ The role of tucotuzumab has not been proven and its effects are still uncertain. Further studies involving a larger population may be appropriate in specific subtypes of patients receiving prior cranial irradiation for prophylaxis.

\section{Trastuzumab}

Human epidermal growth factor receptor 2 (HER2), a member of the HER family, is known to be involved in the signaling pathways which control cell proliferation, differentiation and apoptosis. Trastuzumab, a HER2-targeted agent, exerts an anti-tumor effect by blocking constitutive HER2 signaling, suppressing angiogenesis and by antibodydependent cell-mediated cytotoxicity. The HER2 overexpression was detected in $17.9 \%$ of SCLC patients using IHC, which served as a marker for poor prognosis in extensive-stage SCLC. ${ }^{26,27}$ The chemotherapy-regulated microRNA-125-HER2 pathway as a novel therapeutic target for trastuzumab-mediated cellular cytotoxicity in SCLC seemed to be feasible. ${ }^{28}$ HER2 is upregulated when HER2expressing SCLC cells acquire chemoresistance. A stepwise treatment of these positive cells with trastuzumab and bevacizumab is promising in terms of chemoresistance in SCLC patients. ${ }^{29}$ A study by Kinehara et al. reported on 2 relapsed HER2-positive SCLC patients treated with trastuzumab plus irinotecan, wherein 1 patient achieved PR after the $1^{\text {st }}$ cycle and underwent 6 cycles without disease progression for 4.5 months, and the other patient received 4 cycles and maintained SD for 3.5 months. ${ }^{30}$ Therefore, this drug combination is considered promising and further clinical studies are encouraged in order to confirm the anti-tumor efficacy of trastuzumab in SCLC.

\section{Bec2}

Ganglioside GD3, a cell surface glycosphingolipid antigen, is expressed on the surface of most SCLC tumors, with a limited expression in normal tissues. Ganglioside GD3 may be an appropriate antigenic target for active immunization to eliminate microscopic residual tumors and to enhance survival. Bec2 is an anti-idiotypic antibody that mimics GD3. Fifteen SCLC patients who had completed the standard therapy received a series of 5 intradermal immunizations consisting of $2.5 \mathrm{mg}$ Bec 2 plus Bacillus CalmetteGuérin (BCG) over 10 weeks. The median relapse-free survival for patients with extensive-stage disease was 11 months, whereas for patients with limited-stage disease it was $>47$ months. The immunization of SCLC patients using Bec2 plus BCG was considered safe. ${ }^{31}$ Bec2/BCG was further evaluated in a phase 3 study of adjuvant vaccination in responding patients with limited-disease SCLC. A study conducted by Horn et al. in 515 patients demonstrated no improvement in the survival rate, PFS or quality of life in the vaccinated group. One-third of the vaccinated patients developed a humoral response, and hence showed a significant trend toward prolonged survival $(\mathrm{p}=0.085) .{ }^{4}$ In 2005, Giaccone et al. demonstrated that the primary toxicities of vaccination were transient skin ulcerations and mild flu-like symptoms. ${ }^{32}$ In the study by Bottomley et al., patients in both groups demonstrated significantly impaired scores on the scale of global quality of life at baseline. However, the symptom scores and health-related quality of life showed no statistical difference between the 2 groups. ${ }^{33}$ Therefore, Bec $2 /$ BCG may be suitable for certain SCLC patients, though the patient selection for this treatment requires further study.

\section{Antibody-drug conjugate}

Antibody-drug conjugate is a type of human or humanized monoclonal antibody conjugated with cytotoxic small molecules using chemical linkers. It represents a paradigm shift in chemotherapy. Delta-like protein 3 (DLL3) is highly expressed in approx. $80 \%$ of SCLC cases, but not in normal adult tissues. DLL3 might be associated with the neuroendocrine phenotype and plays an important role in tumorigenesis. Rovalpituzumab tesirine is an antibody-drug 
conjugate comprised of a humanized monoclonal antibody against DLL3, a dipeptide linker and a pyrrolobenzodiazepine dimer toxin. Rudin et al. showed that rovalpituzumab tesirine exhibited single-agent anti-tumor activity and durability in recurrent or refractory SCLC. A dosage ranging from 0.05 to $0.8 \mathrm{mg} / \mathrm{kg}$ of either q3w or q6w was administered to eligible cases among the 74 patients enrolled. Among the 60 eligible patients treated with an active dose, $11(18 \%)$ patients had a confirmed objective response. The proportion of patients with a response was higher among assessable DLL3-high patients $(10$ [38\%] of 26 patients had a confirmed objective response and 23 [88\%] achieved disease control) than among assessable DLL3-low patients (no confirmed objective responses and 4 [50\%] of 8 patients achieved disease control). The most common grade $3+$ toxicities associated with rovalpituzumab tesirine treatment included pleural effusions (8\%), increased lipase (7\%) and thrombocytopenia (11\%). ${ }^{34}$ Rovalpituzumab tesirine treatment shed new light on SCLC and may represent a major therapeutic breakthrough. The toxicity and safety of rovalpituzumab tesirine are a cause for concern, though, warranting further clinical trials.

Trop-2 is a new target for an antibody-drug conjugate due to its elevated expression in solid cancers, including SCLC. Sacituzumab govitecan is a novel antibody-drug conjugate comprised of 7-ethyl-10-hydroxy-camptothecin - the active metabolite of irinotecan - conjugated to an anti-Trop-2 humanized antibody. A phase $1 / 2$ clinical trial is underway in previously-treated metastatic SCLC patients. The trial involves the administration of sacituzumab govitecan 8 or $10 \mathrm{mg} / \mathrm{kg}$ iv. on days 1 and 8 during a 21-day treatment cycle. On an intention-to-treat basis ( $\mathrm{n}=50$ ), the ORR was $14 \%$, the median response duration 5.7 months, median PFS 3.7 months, and median OS 7.5 months. Grade 3+ drug-related toxicities with an incidence rate greater than $5 \%$ included diarrhea (9\%), fatigue (13\%), anemia (6\%), and neutropenia (34\%). ${ }^{35}$ The side effects of sacituzumab govitecan treatment, such as neutropenia, require further study in patients with previouslytreated SCLC.

All SCLC tumor cells express $\mathrm{HuD}$-antigen, a neuronal RNA-binding protein. BW-2 is a novel antibody-toxin compound derived via the assembly of a mouse anti-human-HuD monoclonal antibody onto streptavidin/saporin complexes. A previous study showed that BW-2 was lethal to SCLC at very low concentrations in vitro. Furthermore, it significantly reduced local tumor progression without causing toxicity in a nude mouse model of human SCLC. ${ }^{36}$ Thus, an anti-HuD-based immunotoxin may be an effective alternative for patients with SCLC, prompting the need for further investigation in controlled clinical trials.

Most SCLC tumors expressing CD56 exhibit a poor 5-year survival rate of only $5-10 \%$. CD56 provides a promising therapeutic target for SCLC. Lorvotuzumab mertansine is an antibody-drug conjugate comprised of a humanized monoclonal antibody against CD56, a cleavable disulfide linker and a tubulin-binding maytansinoid DM1. ${ }^{37}$ Lorvotuzumab mertansine was evaluated in a phase 1 study in patients with CD56-positive solid tumors, including SCLC and others; 52 patients were treated with doses of 4-94 $\mathrm{mg} / \mathrm{m}^{2}$ per day. The MTD was $75 \mathrm{mg} / \mathrm{m}^{2}$ and the recommended phase 2 dose was $60 \mathrm{mg} / \mathrm{m}^{2}$. The most common grade 3 or 4 treatment-emergent AEs were dyspnea and hyponatremia (8.2\% each). Responses included 1 CR, 1 clinical CR and 1 unconfirmed PR in Merkel cell carcinoma, and 1 unconfirmed PR in SCLC. Stable disease was recorded in $25 \%$ of the patients who received doses of $\geq 60 \mathrm{mg} / \mathrm{m}^{2}{ }^{38}$ The limited number of patients enrolled in this study warrants further evaluation.

\section{Others}

ROBO1 is a membrane protein that contributes to tumor angiogenesis and metastasis. The 90Y-labeled anti-ROBO1 monoclonal antibody (90Y-anti-ROBO1 IgG) shows an anti-tumor effect against ROBO1-positive tumors. A biodistribution study was conducted by injecting the 111In-labeled anti-ROBO1 monoclonal antibody (111Inanti-ROBO1 IgG) into ROBO1-positive SCLC xenograft mice. A radioimmunotherapy study was conducted to evaluate the anti-tumor effects. The livers, spleens, kidneys, and lungs displayed a high accumulation of 111In-labeled anti-ROBO1, whereas 90Y-anti-ROBO1 IgG significantly reduced the tumor volume. ${ }^{39}$ Radioimmunotherapy with 90Y-anti-ROBO1 IgG is a promising approach against SCLC, and clinical trials are needed.

Tumor necrosis treatment (TNT) involves the use of degenerating tumor cells and necrotic regions of tumors as targets for radioimmunotherapy. (131)I-chTNT, a chimeric monoclonal antibody labeled with the radionuclide iodine-131, has been approved for advanced lung cancer treatment in China. Eligible patients $(n=107)$ included those with advanced lung cancer refractory to radiotherapy or chemotherapy, and treated with asystemic or intra-tumoral injection of (131)I-chTNT. The results showed an ORR of $34.6 \%$ for all patients and for $50 \%$ of the 10 patients with SCLC. ${ }^{40}$ The adverse side effects were mild and reversible. Despite the high ORR, the limited sample size warrants the need for a clinical trial with a larger SCLC population in order to validate the therapeutic effect and toxicities.

Lewis Y, CD174, is a blood group antigen robustly expressed on the surface of SCLC, and is a potential target for antibody-based immunotherapy. Hu3S193 is an antiLewis Y antibody with superior specificity, affinity and cytotoxicity. Hu3S193 was used to treat 10 progressive SCLC patients -5 with $10 \mathrm{mg} / \mathrm{m}^{2}$ and the remainder with $20 \mathrm{mg} / \mathrm{m}^{2}$. Nine out of the 10 patients completed the course of 4 injections. All fluorodeoxyglucose (FDG)-avid lesions measuring over $2 \mathrm{~cm}$ were visualized using antibody single-photon emission computed tomography. Toxicities included grade 2 urticaria $(n=1)$, grade 1 vomiting $(n=2)$ 
Table 1. Clinical studies antibody therapeutics in small-cell lung cancer with promising results

\begin{tabular}{|c|c|c|c|c|}
\hline Author & $\begin{array}{l}\text { Phase } \\
\text { of clinical trial }\end{array}$ & Study population & $\begin{array}{l}\text { Number } \\
\text { of patients }\end{array}$ & Treatment \\
\hline Ott et al. ${ }^{5}$ & phase $1 b$ & $\begin{array}{c}\text { failed or were unable to receive standard } \\
\text { therapy for SCLC patients }\end{array}$ & 147 & pembrolizumab \\
\hline Antonia et al. ${ }^{6}$ & phase $1 / 2$ & recurrent SCLC & 216 & nivolumab VS nivolumab plus ipilimumab \\
\hline Tiseo et al. ${ }^{14}$ & phase 3 & first-line treatment in extensive-stage SCLC & 204 & $\begin{array}{c}\text { cisplatin-etoposide vs cisplatin-etoposide } \\
\text { plus bevacizumab }\end{array}$ \\
\hline Petrioli et al. ${ }^{15}$ & $\begin{array}{l}\text { single } \\
\text { institution } \\
\text { experience }\end{array}$ & first-line treatment in extensive-stage SCLC & 22 & $\begin{array}{l}\text { cisplatin-etoposide and bevacizumab } \\
\text { followed by oral etoposide and bevacizumab } \\
\text { maintenance }\end{array}$ \\
\hline Mountzios et al. ${ }^{17}$ & phase 2 & chemoresistant relapsed SCLC & 30 & paclitaxel plus bevacizumab \\
\hline Chiang et al. ${ }^{23}$ & phase $1 b$ & untreated extensive-stage SCLC & 27 & $\begin{array}{l}\text { tarextumab in combination with cisplatin } \\
\text { and etoposide }\end{array}$ \\
\hline Bao et al. ${ }^{24}$ & phase 1 & SCLC patients & 9 & MEDI0639 \\
\hline Rudin et al. ${ }^{34}$ & phase 1 & recurrent or refractory SCLC & 74 & rovalpituzumab tesirine \\
\hline Gray et al. ${ }^{35}$ & phase $1 / 2$ & previously treated metastatic SCLC & 31 & sacituzumab govitecan \\
\hline Shah et al. ${ }^{38}$ & phase 1 & $\begin{array}{l}\text { CD56-positive solid tumors, including SCLC, } \\
\text { Merkel cell carcinoma and ovarian cancer }\end{array}$ & 52 & lorvotuzumab mertansine \\
\hline Chen et al. ${ }^{40}$ & pivotal study & $\begin{array}{l}\text { lung cancer failure prior to radiotherapy or } \\
\text { chemotherapy, including SCLC }\end{array}$ & 107 & (131)I-chTNT \\
\hline Krug et al. ${ }^{41}$ & pilot trial & progressive SCLC & 10 & Lewis $Y$ \\
\hline
\end{tabular}

and grade 2 hypertension $(\mathrm{n}=1)$ after the high-dose infusion. ${ }^{41}$ The strong tumor targeting of all FDG-avid lesions measuring over $2 \mathrm{~cm}$ using hu3S193 and visualized under antibody single-photon emission computed tomography warrants further clinical study of SCLC.

Table 2. Drugs with targeted proteins and genes

\begin{tabular}{|l|c|}
\hline \multicolumn{1}{|c|}{ Drugs } & Targeted proteins and genes \\
\hline Pembrolizumab & PD-1 \\
\hline Nivolumab & PD-1 \\
\hline Ipilimumab & CTLA-4 \\
\hline Bevacizumab & VEGF \\
\hline R1507 & IGF-1R \\
\hline Tarextumab & Notch2 and Notch3 \\
\hline MEDI0639 & DLL4 \\
\hline Tucotuzumab & EpCAM \\
\hline Trastuzumab & HER2 \\
\hline Bec2 & GD3 \\
\hline Rovalpituzumab tesirine & DLL-3 \\
\hline Sacituzumab govitecan & Trop-2 \\
\hline BW-2 & HuD-antigen \\
\hline Lorvotuzumab mertansine & CD56 \\
\hline 90Y-anti-ROBO1 lgG & ROBO1 \\
\hline (131)l-chTNT & tumor proliferating cell nuclear antigen \\
\hline hu3S193 & Lewis Y \\
\hline 111ln-hu3S193 & Lewis Y \\
\hline
\end{tabular}

\section{Conclusions}

All the studies to date support the following findings:

- the clinical efficacy of pembrolizumab, nivolumab, ipilimumab, and rovalpituzumab tesirine in SCLC showed promising results and called for further verification through phase 3 clinical trials;

- chemotherapy combined with bevacizumab in SCLC can improve PFS;

- the therapeutic effect of tarextumab, sacituzumab govitecan, BW-2, and lorvotuzumab mertansine in SCLC requires further evaluation;

- clinical studies are encouraged to confirm the antitumor efficacy of figitumumab and trastuzumab in SCLC;

- Bec2/BCG as adjuvant vaccination in responding patients with limited-disease SCLC showed no improvement in the survival, PFS or quality of life.

Clinical studies regarding antibody therapeutics in SCLC with promising results are presented in Table 1 . The drugs investigated in SCLC with targeted proteins and genes are shown in Table 2.

\section{References}

1. Siegel R, Naishadham D, Jemal A. Cancer statistics, 2013. CA Cancer J Clin. 2013;63:11-30.

2. Chen W, Zheng R, Baade PD, et al. Cancer statistics in China, 2015. CA Cancer J Clin. 2016;66:115-132.

3. Govindan R, Page N, Morgensztern D, et al. Changing epidemiology of small-cell lung cancer in the United States over the last 30 years: Analysis of the surveillance, epidemiologic, and end results database. J Clin Oncol. 2006;24:4539-4544.

4. Horn L, Reck M, Spigel DR. The future of immunotherapy in the treatment of small cell lung cancer. Oncologist. 2016;21:910-921. 
5. Ott PA, Elez E, Hiret $\mathrm{S}$, et al. Pembrolizumab in patients with extensive-stage small-cell lung cancer: Results from the phase Ib KEYNOTE-028 study. J Clin Oncol. 2017;35:3823-3829.

6. Antonia SJ, López-Martin JA, Bendell J, et al. Nivolumab alone and nivolumab plus ipilimumab in recurrent small-cell lung cancer (CheckMate 032): A multicentre, open-label, phase 1/2 trial. Lancet Oncol. 2016;17:883-895.

7. Berghoff AS, Ricken G, Wilhelm D, et al. Tumor infiltrating lymphocytes and PD-L1 expression in brain metastases of small cell lung cancer (SCLC). J Neurooncol. 2016;130:19-29.

8. Schalper KA, Carvajal-Hausdorf DE, McLaughlin JF, et al. Objective measurement and significance of PD-L1, B7-H3, B7-H4 and TILs in small cell lung cancer (SCLC). J Clin Oncol. 2016;34(Suppl abstr):8566.

9. Miao L, Lu Y, Xu Y, et al. PD-L1 and c-MET expression and survival in patients with small cell lung cancer. Oncotarget. 2017;8:5397853988.

10. Takada K, Toyokawa G, Okamoto T, et al. An immunohistochemical analysis of PD-L1 protein expression in surgically resected small cell lung cancer using different antibodies and criteria. Anticancer Res. 2016;36:3409-3412.

11. Roviello G, Generali D. Is there a place for bevacizumab in patients with extensive-stage small cell lung cancer? Curr Cancer Drug Targets. 2016;16:209-214.

12. Ustuner Z, Saip P, Yasasever V, et al. Prognostic and predictive value of vascular endothelial growth factor and its soluble receptors, VEGFR-1 and VEGFR-2 levels in the sera of small cell lung cancer patients. Med Oncol. 2008;25:394-399.

13. Pujol JL, Lavole A, Quoix E; French Cooperative Thoracic Intergroup IFCT: Randomized phase II-III study of bevacizumab in combination with chemotherapy in previously untreated extensive small-cell lung cancer: Results from the IFCT-0802 trial. Ann Oncol. 2015;26:908-914.

14. Tiseo M, Boni L, Ambrosio F, et al. Italian, multicenter, phase III, randomized study of cisplatin plus etoposide with or without bevacizumab as first-line treatment in extensive-disease smallcell lung cancer: The GOIRC-AIFA FARM6PMFJM trial. J Clin Oncol. 2017;35:1281-1287.

15. Petrioli R, Roviello G, Laera L, et al. Cisplatin, etoposide, and bevacizumab regimen followed by oral etoposide and bevacizumab maintenance treatment in patients with extensive-stage small cell lung cancer: A single-institution experience. Clin Lung Cancer. 2015;16: 229-234.

16. Jalal S, Bedano P, Einhorn L, et al. Paclitaxel plus bevacizumab in patients with chemosensitive relapsed small cell lung cancer: A safety, feasibility, and efficacy study from the Hoosier Oncology Group. J Thorac Oncol. 2010;5:2008-2011.

17. Mountzios G, Emmanouilidis C, Vardakis N, et al. Paclitaxel plus bevacizumab in patients with chemoresistant relapsed small cell lung cancer as salvage treatment: A phase II multicenter study of the Hellenic Oncology Research Group. Lung Cancer. 2012;77:146-150.

18. Gately K, Collins I, Forde L, et al. A role for IGF-1R-targeted therapies in small-cell lung cancer? Clin Lung Cancer. 2011;12:38-42.

19. Ferté $C$, Loriot $Y$, Clémenson $C$, et al. IGF-1R targeting increases the antitumor effects of DNA-damaging agents in SCLC model: An opportunity to increase the efficacy of standard therapy. Mol Cancer Ther. 2013;12:1213-1222.

20. Cao H, Dong W, Shen $\mathrm{H}$, et al. Combinational therapy enhances the effects of anti-IGF-1R mAb figitumumab to target small cell lung cancer. PLoS One. 2015;10:0135844.

21. Previs RA, Coleman RL, Harris AL, Sood AK. Molecular pathways: Translational and therapeutic implications of the Notch signaling pathway in cancer. Clin Cancer Res. 2015; 21:955-961.

22. Yen WC, Fischer MM, Axelrod F, et al. Targeting Notch signaling with a Notch2/Notch3 antagonist (tarextumab) inhibits tumor growth and decreases tumor-initiating cell frequency. Clin Cancer Res. 2015;21: 2084-2095.

23. Chiang AC, Rudin CM, Spira Al, et al. Updated results of phase $1 \mathrm{~b}$ study of tarextumab (TRXT, anti-Notch2/3) in combination with etoposide and platinum (EP) in patients (pts) with untreated extensivestage small-cell lung cancer (ED-SCLC). J Clin Oncol. 2016;34(Suppl abstr):8564.
24. Bao $\mathrm{H}$, Chen $\mathrm{X}$, Thomas $\mathrm{S}$, et al. Evaluation of anti-cancer stem cell activity of the anti-DLL4 antibody MEDI0639 in a phase I clinical trial of SCLC. J Clin Oncol. 2016;34(Suppl abstr):20093.

25. Gladkov O, Ramlau R, Serwatowski P, et al. Cyclophosphamide and tucotuzumab (huKS-IL2) following first-line chemotherapy in responding patients with extensive-disease small-cell lung cancer. Anticancer Drugs. 2015;26:1061-1068.

26. Potti A, Willardson J, Forseen $C$, et al. Predictive role of HER-2/neu overexpression and clinical features at initial presentation in patients with extensive stage small cell lung carcinoma. Lung Cancer. 2002; 36:257-261.

27. Canoz O, Ozkan M, Arsav V, et al. The role of c-erbB-2 expression on the survival of patients with small-cell lung cancer. Lung. 2006;184: 267-272.

28. Yagishita S, Fujita Y, Kitazono S, et al. Chemotherapy-regulated microRNA-125-HER2 pathway as a novel therapeutic target for trastuzumab-mediated cellular cytotoxicity in small cell lung cancer. $\mathrm{Mol}$ Cancer Ther. 2015;14:1414-1423.

29. Minami T, Kijima T, Kohmo S, et al. Overcoming chemoresistance of small-cell lung cancer through stepwise HER2-targeted antibodydependent cell-mediated cytotoxicity and VEGF-targeted antiangiogenesis. Sci Rep. 2013;3:2669.

30. Kinehara Y, Minami T, Kijima T, et al. Favorable response to trastuzumab plus irinotecan combination therapy in two patients with HER2-positive relapsed small-cell lung cancer. Lung Cancer. 2015;87: 321-325.

31. Grant SC, Kris MG, Houghton AN, Chapman PB. Long survival of patients with small cell lung cancer after adjuvant treatment with the anti-idiotypic antibody BEC2 plus Bacillus Calmette-Guérin. Clin Cancer Res. 1999;5:1319-1323.

32. Giaccone G, Debruyne C, Felip E, et al. Phase III study of adjuvant vaccination with Bec2/bacille Calmette-Guerin in responding patients with limited-disease small-cell lung cancer (European Organisation for Research and Treatment of Cancer 08971-08971B; Silva Study). J Clin Oncol. 2005;23:6854-6864.

33. Bottomley A, Debruyne C, Felip E, et al. Symptom and quality of life results of an international randomised phase III study of adjuvant vaccination with Bec2/BCG in responding patients with limited disease small-cell lung cancer. Eur J Cancer. 2008;44:2178-2184.

34. Rudin CM, Pietanza MC, Bauer TM, et al. Rovalpituzumab tesirine, a DLL3-targeted antibody-drug conjugate, in recurrent small-cell lung cancer: A first-in-human, first-in-class, open-label, phase 1 study. Lancet Oncol. 2017;18(1):42-51.

35. Gray JE, Heist RS, Starodub AN, et al. Therapy of small cell lung cancer (SCLC) with a topoisomerase-l-inhibiting antibody-drug conjugate (ADC) targeting Trop-2, sacituzumab govitecan. Clin Cancer Res. 2017;23:5711-5719.

36. Ehrlich D, Wang B, Lu W, Dowling P, Yuan R. Intratumoral anti-HuD immunotoxin therapy for small cell lung cancer and neuroblastoma. J Hematol Oncol. 2014;7:91.

37. Whiteman KR, Johnson HA, Mayo MF, et al. Lorvotuzumab mertansine, a CD56-targeting antibody-drug conjugate with potent antitumor activity against small cell lung cancer in human xenograft models. Mabs. 2014;6:556-566.

38. Shah MH, Lorigan P, O'Brien ME, et al. Phase I study of IMGN901, a CD56-targeting antibody-drug conjugate, in patients with CD56positive solid tumors. Invest New Drugs. 2016;34:290-299.

39. Fujiwara K, Koyama K, Suga K, et al. 90Y-labeled anti-ROBO1 monoclonal antibody exhibits antitumor activity against small cell lung cancer xenografts. PLoS One. 2015;10:e0125468.

40. Chen S, Yu L, Jiang C, et al. Pivotal study of iodine-131-labeled chimeric tumor necrosis treatment radioimmunotherapy in patients with advanced lung cancer. J Clin Oncol. 2005;23:1538-1547.

41. Krug LM, Milton DT, Jungbluth AA, et al. Targeting Lewis $Y(L e(y))$ in small cell lung cancer with a humanized monoclonal antibody, hu3S193: A pilot trial testing two dose levels. J Thorac Oncol. 2007;2: 947-952. 